\title{
Die Übernahme-Richtlinie und der Mindestpreis des Pflichtangebots
}

\author{
MARTIN WINNER
}

\section{Einleitung}

In seinem grundlegenden Beitrag zum europäischen Übernahmerecht, der in Zusammenhang mit der Revision der Übernahme-Richtlinie ${ }^{1}$ im Jahre 2012 entstanden ist, nimmt Klaus J. Hopt auch zu Fragen des Mindestpreises für ein Pflichtangebot Stellung. ${ }^{2}$ Er sieht in der Umsetzung der entsprechenden Vorgaben der Richtlinie ein Paradebeispiel für gold plating und hält insbesondere fest, dass Mitgliedstaaten die Möglichkeit für die Preisanpassung durch die Aufsichtsbehörden völlig unterschiedlich ausgenutzt haben. Im Ergebnis spricht sich der Jubilar - vor allem angesichts der bestehenden Unsicherheit über die „richtige“ Regelung - dafür aus, den diesbezüglichen Regelungsspielraum für die Mitgliedstaaten bei einer etwaigen Revision der ÜbRL nicht einzuschränken.

Der folgende Beitrag geht angesichts kürzlich abgeschlossener ${ }^{3}$ und seit Ende 2019 laufender ${ }^{4}$ Verfahren vor dem EuGH der Frage nach, ob dieser Befund weiterhin Bestand haben kann. Denn gerade die Frage der richtigen Berechnung der bei einem Pflichtangebot mindestens zu bietenden Gegenleistung beschäftigt das europäische Höchstgericht zusehends. Ganz generell fällt auf, dass in jüngster Zeit übernahmerechtliche Fragen erstmals in einer gewissen Häufung den Gerichtshof erreichen; ${ }^{5}$ ob es dafür besondere Gründe gibt, wäre noch gesondert zu untersuchen.

1 RL 2004/25/EG v. 21.4.2004 betreffend Übernahmeangebote, ABl. L 142 v. 30.4. 2004, S. 12 (im Folgenden: ÜbRL).

${ }^{2}$ Hopt, Europäisches Übernahmerecht, 2013, S. $57 \mathrm{ff}$.

3 EuGH v. 20.7.2017, Marco Tronchetti Provera SpA et al v Consob, C-206/16, ECLI:EU:C:2017:572. Siehe aber auch schon EFTA-Gerichtshof v. 10.12.2010, Periscopus AS ./. Oslo Bors ASA und Erika Must AS, E-1/10, [2009-2010] EFTA Ct. Rep. 198.

${ }^{4}$ C-735/19 Euromin Holdings (Cyprus) Limited v Finanšu un kapitāla tirgus komisija, anhängig seit 7.10.2019 (Lettland).

${ }^{5}$ Neben dem in der vorigen Fußnote genannten Verfahren: C-339/19 SC Romenergo SA/Aris Capital SA v Autoritatea de Supraveghere Financiara, anhängig seit 25.4.2019 (Rumänien; Definition des gemeinsamen Vorgehens) und C-546/18 Adler Real Estate u.a., anhängig seit 23.8.2018 (Österreich; Übernahmeaufsicht und fair trial). 
Der Beitrag beschäftigt sich im Kern mit der Frage der Vorerwerbe, wobei viele Aussagen grundsätzlich auch für Paralleltransaktionen gem. Art. 5 Abs. 4 Satz 2 ÜbRL oder für die - teilweise nach den nationalen Übernahmerechten zu berücksichtigenden - Nacherwerbe ${ }^{6}$ Geltung beanspruchen. Dabei geht es, nach einer kurzen Einführung in die Rechtslage, zunächst um die richtige Feststellung der Gegenleistung, die der Bieter für die angebotsgegenständlichen Wertpapiere erbracht hat; das hängt eng mit den Möglichkeiten für nationale Behörden zusammen, den so ermittelten Mindestpreis nach oben oder nach unten anzupassen. Den Abschluss bildet die (wieder) aktuelle Frage, ob der Börsenkurs als jedenfalls zu beachtende Mindestschwelle angesichts der ÜbRL Bestand haben kann.

\section{Die Richtlinien-Regelung zum Mindestpreis}

Die einschlägige Vorschrift ist Art. 5 Abs. 4 ÜbRL. Sie gilt allerdings nur für Pflichtangebote als Rechtsfolge der Kontrollerlangung, nicht aber für Übernahmeangebote, mit denen die Kontrolle erlangt werden soll; $;$ gerade dies war im Zusammenhang mit dem low balling, also Angeboten zu unattraktiven Bedingungen, mit denen die Kontrollschwelle möglichst billig überschritten werden soll, ein Thema, ${ }^{8}$ was hier aber außen vor bleiben soll. Gerade Deutschland ${ }^{9}$ und Österreich ${ }^{10}$ haben die Regelungen zum Mindestpreis - in Einklang mit dem von der Richtlinie verfolgten Konzept der Mindestharmonisierung (Art. 3 Abs. 2 Buchstabe b ÜbRL) - auf freiwillige Angebote erstreckt.

Nach Art. 5 Abs. 4 UAbs. 1 ÜbRL muss der Bieter im Pflichtangebot den höchsten Preis zahlen, der von ihm oder einer mit ihm gemeinsam handelnden Person in einem Referenzzeitraum von - nach Wahl der Mitgliedstaaten - sechs bis zwölf Monaten vor dem Angebot für die Wertpapiere gezahlt worden ist. Die ÜbRL lässt offen, wie der Preis bestimmt wird, den der Bieter gezahlt hat. Das ist beim Barerwerb unproblematisch, aber dann schwierig, wenn die Kontrollschwelle durch einen Aktientausch überschritten wird; denn dann muss diese Gegenleistung des Bieters bewertet werden, um

${ }^{6}$ Siehe $₫ 31$ Abs. 4 WpÜG und $\S 16$ Abs. 7 österreichisches Übernahmegesetz (im Folgenden öÜbG), öBGBl. I 1998/127.

7 Siehe schon den unter maßgeblicher Beteiligung des Jubilars vorgelegten Report of the High Level Group of Company Law Experts on Issues Related to Takeover Bids, 2002, S. 45 (https://papers.ssrn.com/sol3/papers.cfm?abstract_id=315322).

${ }^{8}$ Dazu Hopt, Europäisches Übernahmerecht (Fn. 2) S. 46ff.; Tyrolt/Cascante in: Mülbert/Kiem/Wittig, 10 Jahre WpÜG, 2011, S. 110, $140 \mathrm{ff}$.

${ }^{9}$ Vgl. die Platzierung von $₫ 31$ WpÜG im Abschnitt über „Übernahmeangebote“.

10 Vgl. § 26 Abs. 1 öÜbG. 
den Angebotspreis in bar zu bestimmen. ${ }^{11}$ Soweit der Bieter allerdings auch im Angebot den Adressaten diejenigen Wertpapiere bietet oder bieten muss $^{12}$, die bei der Transaktion, mit der die Kontrolle erworben wurde, geleistet wurden, genügt es für die Gleichbehandlung dem Grundsatz nach, diese Wertpapiere - ohne nähere Bewertung - im selben (Umtausch)Verhältnis zu bieten. Damit ist die Bewertungsfrage zwar nicht vom Tisch, sofern immer noch wahlweise eine bare Gegenleistung geboten werden muss, ${ }^{13}$ sie ist aber doch entschärft. Daneben gibt es noch zahlreiche andere Konstellationen, in denen die vom Bieter für die angebotsgegenständlichen Wertpapiere erbrachte Leistung nicht ohne weiteres festzumachen ist und die uns in der Folge beschäftigten werden.

Allerdings ist diese gewährte Gegenleistung nicht in allen Fällen auch zwingend der Mindestpreis für das Pflichtangebot. Denn Art. 5 Abs. 4 UAbs. 2 ÜbRL erlaubt es den Mitgliedstaaten, ihre Aufsichtsstellen zu „ermächtigen, den in Unterabsatz 1 genannten Preis unter ganz bestimmten Voraussetzungen und nach eindeutig festgelegten Kriterien abzuändern,“ wenn die allgemeinen Grundsätze gem. Art. 3 Abs. 1 ÜbRL eingehalten werden. Allerdings sind die Mitgliedstaaten nicht verpflichtet, solche Ausnahmen vorzusehen; gerade Deutschland hat von der Option zumindest explizit nicht Gebrauch gemach ${ }^{14}$. Die Vorschrift ist lex specialis zu Art. 4 Abs. 5 UAbs. 2 ÜbRL,,$^{15}$ der generell festhält, dass und unter welchen Voraussetzungen Abweichungen von Vorschriften der Richtlinie zulässig sind.

Der Wortlaut von Art. 5 Abs. 4 UAbs. 2 ÜbRL verlangt eine Determinierung sowohl der Voraussetzungen für die Abweichung als auch der dann zu berücksichtigenden Kriterien; ${ }^{16}$ es bleibt lediglich offen, ob diese Determinierung durch die Mitgliedstaaten selbst erfolgen muss oder auch durch die Aufsichtsbehörden erfolgen kann, wobei letzteres richtig sein dürfte. Damit steht freilich in einem Spannungsverhältnis, dass nach den beiden folgenden Sätzen die Mitgliedstaaten Listen über die Voraussetzungen für ein Abweichen und Festlegungen über die heranzuziehenden Kriterien erlassen „können“. Das könnte man so verstehen, dass die genaue Determinierung doch

${ }_{11}$ Dazu z.B. Winner, $\$ 23$ Unternehmensbewertung im Übernahmerecht, in: Fleischer/ Hüttemann, Rechtshandbuch Unternehmensbewertung, 2. Aufl. 2019, Rn. $23.105 \mathrm{ff}$.

12 Siehe Rule 11.2 City Code on Takeovers and Mergers (wenn mehr als 10\% der vom Angebot erfassten Wertpapiere innerhalb der letzten drei Monate vor dem Angebot im Tausch gegen solche Wertpapiere erworben wurden); dazu Kershaw, Principles of Takeover Regulation, 2016, Rn. 6.42.

${ }^{13}$ Siehe $₫ 31$ Abs. 3 WpÜG; strenger (jedenfalls Barangebot erforderlich) $₫ 25 \mathrm{~b}$ Abs. 2 öÜbG.

${ }^{14}$ Siehe Lutter/Bayer/J. Schmidt, Europäisches Unternehmens- und Kapitalmarktrecht, 6. Aufl. 2017, Rn. 28.55.

${ }^{15}$ Lutter/Bayer/J. Schmidt (Fn. 14) Rn. 28.54.

16 Anders aber Lutter/Bayer/J. Schmidt (Fn. 14) Rn. 28.54: Voraussetzungen „und/ oder" Kriterien. 
nicht erforderlich ist, oder aber - richtigerweise - so, dass sie entweder durch die Mitgliedstaaten oder durch die Aufsichtsbehörden erfolgen kann. Gute Legistik ist das nicht.

Art. 5 Abs. 4 UAbs. 2 ÜbRL enthält in den Sätzen 2 und 3 demonstrative Aufzählungen von Kriterien, bei deren Vorliegen der Preis korrigiert werden darf, und von Kriterien, die in solchen Fällen für die Bestimmung des maßgeblichen Preises heranzuziehen sein können. Nicht alle Kriterien der ersten Gruppe sind leicht zu verstehen: Was soll es bedeuten, dass eine Korrektur möglich sein soll, wenn „der Höchstpreis in einer Vereinbarung zwischen Käufer und Verkäufer gemeinsam festgelegt worden ist“? Passiert das bei einem Vertrag über einen Aktienerwerb, der ja schließlich jeder Referenztransaktion zugrunde liegt, nicht immer? Gemeint ist wohl, dass der gezahlte Preis durch eine solche Vereinbarung (oder mehrere) verschleiert wurde. ${ }^{17}$ Ebenso bleibt unklar, warum wiederholt von „Marktpreisen“ die Rede ist. Denn zu berücksichtigen ist ja nur der bezahlte Preis, aber nicht ein Marktpreis im Sinn von Börsenkursen. Erkennbar geht es den Beispielen für mögliche Voraussetzungen einer Korrektur um zwei unterschiedliche Sachverhalte: einerseits um manipulative Praktiken, andererseits um Situationen, in denen der im Rahmen des Vorerwerbs bezahlte Preis für das Pflichtangebot nicht mehr angemessen ist.

Klarer sind die folgenden Kriterien, nach denen eine Korrektur vorzunehmen ist, namentlich der „durchschnittliche[...] Marktwert während eines bestimmten Zeitraums, de[r] Liquidationswert der Gesellschaft oder andere objektive Bewertungskriterien, die allgemein in der Finanzanalyse verwendet werden". Diese Kriterien sind nur heranzuziehen, wenn eine Abweichung aufgrund der Umstände des Einzelfalls tunlich erscheint - aber nicht generell, was uns noch beschäftigen wird. ${ }^{18}$

Schließlich ist jede Entscheidung der Aufsichtsstellen zur Änderung des angemessenen Preises zu begründen und bekannt zu machen (Art. 5 Abs. 4 UAbs. 3 ÜbRL). Es entspricht der Richtlinie daher nicht, dass die Veröffentlichung einer Angebotsunterlage mit einem abweichenden Preis von der Aufsichtsbehörde bloß nicht untersagt wird. Auch das hat für die deutsche und österreichische Rechtslage Bedeutung, die nun anhand der derzeit einzigen Entscheidung des EuGH zu Preisfragen und einer etwas älteren Entscheidung des EFTA-Gerichtshofs dargelegt wird.

17 Vgl. auch den Bericht der High Level Group (Fn. 7) S. 50, der die entsprechende Ausnahme noch wie folgt und damit viel klarer formuliert: „the highest price was set by collusion (i.e. an agreement with the vendor aimed at evading the highest price paid rule)“.

18 Siehe unten IV. 


\section{Die Rechtssachen Marco Tronchetti Provera $S p A$ und Periscopus}

\section{Die Entscheidungen}

Der Ausgangspunkt der Rechtssache Marco Tronchetti Provera SpA u.a. $\checkmark$ Consob $^{19}$ ist rasch wiedergegeben: Die Verkäuferin, Malacalza Investimenti Srl (MCI) verkaufte 60,99\% der Aktien an Camfin SpA an Marco Tronchetti Provera $S p A(M T P)$ um 0,8 Euro pro Aktie; zu diesem Preis wurde auch ein Pflichtangebot gelegt. Allerdings hielt die Zielgesellschaft Camfin ihrerseits 26,19\% an Pirelli E C. SpA und MCI hatte von zwei anderen Pirelli-Aktionären 6,98\% der Pirelli-Aktien zu 7,8 Euro pro Aktie erworben, obwohl der Wert dieser Aktien bei 8 Euro lag. Weil diese beiden Pirelli-Aktionäre mit Camfin eine Aktionärsvereinbarung über die PirelliAktien abgeschlossen hatten, gingen die italienischen Gerichte davon aus, dass die Minderheitsaktionäre mit der Angebotsgegenleistung nicht denselben Vorteil erhalten hatten wie MCI und dass diese Mehrleistung an MCI der Bieterin MTP zuzurechnen war. Lässt man einmal beiseite, dass sich die genauen Gründe für diese Zurechnung aus dem Urteil nicht erschließen lassen, da nähere Angaben über die Tragweite der Absprache unter den PirelliAktionären und die wechselseitige Interessenlage am Abschluss der CamfinTransaktion fehlen, so ist der Grundgedanke aber doch klar: Der Verkäufer bekommt weniger Geld für die angebotsbetroffenen Aktien und bekommt dafür eine andere Leistung, hier die Aktien an Pirelli, billiger.

Die Consob und in der Folge die italienischen Gerichte passten deswegen die Gegenleistung für das Pflichtangebot nach oben auf 0,83 Euro pro Aktie an. Dabei stützten sie sich auf Vorschriften des italienischen Rechts, die eine Anpassung zulässt, wenn es zu „Kollusion“ zwischen dem Bieter und den Verkäufern gekommen ist. Dabei wurde dieser unbestimmte Rechtsbegriff abweichend $\mathrm{zu}$ anderen Vorschriften des italienischen Rechts ausgelegt: Es genüge die objektive Eignung zur Umgehung der Preisbildungsvorschriften, ohne dass es auf eine subjektive Umgehungsabsicht ankomme.

Dem EuGH wurde vorgelegt, ob der Begriff „Kollusion“ ausreichend determiniert ist; denn Art. 5 Abs. 4 UAbs. 2 ÜbRL verlangt, dass die Aufsichtsstellen den Preis „unter ganz bestimmten Voraussetzungen“ anpassen können, was auch so verstanden werden kann, dass an die Bestimmtheit solcher Normen ganz besonders hohe Anforderungen zu stellen sind. Eine solche Auslegung war vor der Entscheidung des EuGH besonders nahe liegend, hatte doch der EFTA-Gerichtshof in seiner Periscopus-Entschei-

19 EuGH v. 20.7.2017, Marco Tronchetti Provera SpA et al v Consob, C-206/16, ECLI:EU:C:2017:572. 
dung $^{20}$ im Jahr 2010 festgehalten, dass eine norwegische Vorschrift, nach welcher der Preis des Pflichtangebots zumindest dem Marktpreis zum Zeitpunkt, zu dem die Angebotspflicht ausgelöst wird, entsprechen musste, wenn dieser „klar“ über dem Vorerwerbspreis liegt, für Zwecke von Art. 5 Abs. 4 UAbs. 2 ÜbRL nicht ausreichend determiniert ist. Vielmehr wäre es erforderlich gewesen, nähere Berechnungsmethoden für die Bestimmung dieses Marktpreises aufzunehmen. Allerdings hatte auch der EFTA-Gerichtshof festgehalten, dass es nicht dem - in Erwägungsgrund (6) ÜbRL ausdrücklich festgehaltenen - Prinzip der Flexibilität entsprechen würde, wenn die Mitgliedstaaten jede spezifische Ausnahmesituation im Detail ex ante vorzeichnen müssten. ${ }^{21}$

In Sachen Marco Tronchetti Provera war der EuGH - aus Sicht der nationalen Gesetzgeber - deutlich liberaler als der EFTA-Gerichtshof in seiner Periscopus-Entscheidung. Denn der EuGH hielt fest, dass die ÜbRL nicht verlange, dass „eine einen abstrakten Rechtsbegriff verwendende Rechtsvorschrift die verschiedenen konkreten Fälle nennen muss, in denen sie Anwendung finden kann, da der Gesetzgeber nicht jeden dieser Fälle im Voraus bestimmen kann. "22 Daher genüge die Verwendung des Begriffs „Kollusion“, sofern „sich die Auslegung eines solchen Begriffs im Bereich der Übernahmeangebote anhand der vom innerstaatlichen Recht anerkannten Auslegungsmethoden hinreichend klar, bestimmt und voraussehbar aus der in Rede stehenden nationalen Regelung ableiten lässt" ${ }^{23}$ Damit hat der EuGH die Frage im Ergebnis an die nationalen Gerichte zurückgespielt. Unter welchen Voraussetzungen nationale Regeln „hinreichend“ bestimmt sind, ist damit freilich nicht beantwortet.

\section{Ermittlung oder Anpassung der Gegenleistung?}

Hat der EuGH aber das Thema überhaupt richtig erfasst? Denn geht es wirklich um eine Anpassung der Gegenleistung des Pflichtangebots im Sinne von Art. 5 Abs. 4 UAbs. 2 ÜbRL? Genauso gut könnte man das Problem nämlich auch anders einordnen, nämlich als (vorgelagerte) Frage der richtigen Ermittlung der im Rahmen des Vorerwerbs erbrachten Leistung. ${ }^{24}$ Mit

${ }^{20}$ EFTA-Gerichtshof v. 10.12.2010, Periscopus AS ./. Oslo Bors ASA und Erika Must AS, E-1/10, [2009-2010] EFTA Ct. Rep. 198.

${ }^{21} \mathrm{AaO}$ Rn. 47.

22 EuGH v. 20.7.2017, Marco Tronchetti Provera SpA et al v Consob, C-206/16, ECLI: EU:C:2017:572, Rn. 42.

${ }^{23} \mathrm{AaO}$ Rn. 46.

${ }^{24}$ Ebenso wäre es möglich, solche versteckten Verbesserungen in gesonderten Vereinbarungen zwischen Bieter und verkaufendem Aktionär schlichtweg zu verbieten, sofern die Aufsichtsbehörde ihre Zustimmung nicht gibt. Das ist der Ansatz von Rule 16.1 City Code on Takeovers and Mergers; dazu Kershaw, Principles (Fn. 12) Rn. 6.32f. 
anderen Worten hat $M C I$ nach diesem Verständnis nicht 0,8 Euro, sondern in Summe 0,83 Euro pro Aktie erhalten. Dieser Vorteil ist nach diesem Ansatz an die Minderheitsaktionäre weiterzugeben, ohne dass noch eine gesonderte Anpassung erforderlich wäre.

Dieser Zugang entspricht dem österreichischen Recht. Denn $₫ 26$ Abs. 3 Satz 1 öÜbG verlangt, dass bei der Ermittlung des Gesamtwerts auch weitere zugewendete oder zugesagte Zahlungen oder sonstige vermögenswerte Vorteile einzubeziehen sind, wenn diese in wirtschaftlichem Zusammenhang mit der erlangten kontrollierenden Beteiligung stehen; die nachgelagerte Anpassung dieses so ermittelten Preises wird dann im Folgesatz der Vorschrift geregelt. ${ }^{25}$ Fälle aus der Entscheidungspraxis betreffen z.B.:26 den parallelen Erwerb von (durch die Zielgesellschaft weiterhin zu nutzenden) Markenrechten durch den Bieter von der früheren Konzernherrin, wofür es nach Ansicht der Übernahmekommission auf die Angemessenheit der vereinbarten Gegenleistung ankommt; die gesonderte Abgeltung eines Wettbewerbsverbots der veräußernden Aktionäre (jedenfalls preiserhöhend ${ }^{27}$ ); Übernahme der Rechtsberatungskosten der Verkäuferin durch den Bieter (jedenfalls preiserhöhend) $;^{28}$ die gesonderte Übernahme von Patronatserklärungen bzw. Stützungsvereinbarungen der bisherigen Kontrollaktionärin durch den Bieter und damit die Befreiung von einer Verbindlichkeit (im konkreten Fall aufgrund der Ausgestaltung nicht preiserhöhend) ${ }^{29}$. Auch eine Konstellation wie in der Entscheidung Marco Tronchetti Provera wäre ohne jeden Zweifel auf Basis dieser Vorschrift zu behandeln gewesen.

Die Rechtslage zu \31 WpÜG bzw. \4 WpÜG-AngVO ist im Ergebnis vergleichbar, wenngleich ausdrückliche gesetzliche Anordnungen fehlen:30 Zusätzliche Leistungen des Bieters (samt gemeinsam vorgehender Rechtsträger) an den Veräußerer sind dann werterhöhend, wenn sie in einem Austauschzusammenhang mit dem Aktienerwerb stehen. Das wird insbesondere bei Parallelgeschäften mit überhöhter Leistung auf Seiten des Bieters angenommen, also wiederum der Fall, welcher der Entscheidung Marco Tron-

${ }^{25}$ Dazu Diregger/Kalss/Winner, Das österreichische Übernahmerecht, 2. Aufl. 2007, Rn. 303.

26 Siehe auch die Beispiele bei Huber in: Huber, Übernahmegesetz, 2. Aufl. 2016, $\$ 26$ Rn. 51.

${ }_{27}$ Anders für Deutschland Noack in: Schwark/Zimmer, Kapitalmarktrechtskommentar, 4. Aufl. 2010, § 31 WpÜG Rn. 29.

${ }_{28}$ Dies alles in Übernahmekommission v. 3.12.2014, GZ 2004/2/3-55, Hirsch Servo AG (alle Entscheidungen abrufbar unter www.takeover.at/entscheidungen/).

29 Übernahmekommission v. 17.12.2001, GZ 2001/2/3-395, Lauda Air.

30 Siehe z.B. mit Unterschieden in Details Krause in: Assmann/Pötzsch/Schneider, WpÜG, 2. Aufl. 2013, 』31 Rn. 113 ff.; Kremer/Oesterbaus in: Kölner Kommentar zum WpÜG, 2. Aufl. 2010, \31 Rn. 71 ff.; Noack in: Schwark/Zimmer (Fn. 27) \31 WpÜG Rn. 28; Reinhardt/Kocher in: Paschos/Fleischer, Handbuch Übernahmerecht nach dem WpÜG, 2017, § 15 Rn. 156; Tyrolt/Cascante in: Mülbert/Kiem/Wittig (Fn. 8) S. $128 \mathrm{ff}$. 
chetti Provera zugrunde lag. Das entspricht nicht nur der ganz herrschenden Ansicht, sondern auch der Praxis der BaFin. ${ }^{31}$

Angesichts dieser Ausgangsbasis ist es nur konsequent, dass die für Zwecke der Preisfindung maßgebliche Gegenleistung gegenüber dem im Erwerbsvertrag festgehaltenen Kaufpreis reduziert wird, wenn der Veräußerer noch weitere Leistungen erbringt, die nicht mit dem Erwerb der Aktie selbst in Zusammenhang stehen. ${ }^{32}$ In Österreich werden die entsprechenden Konstellationen (z.B. besondere Haftungen des Veräußerers oder dem Erwerber ersetzte Konventionalstrafen) interessanterweise aber unter dem Topos „Anpassung der Gegenleistung" diskutiert. ${ }^{33}$ Unabhängig von der Einordnung steht diese Ansicht in Einklang mit Art. 5 Abs. 4 UAbs. 2 ÜbRL, der eine Korrektur „nach oben oder nach unten“ zulässt. Freilich ist die Missbrauchsgefahr hier besonders hoch, ${ }^{34}$ ebenso wie die Abgrenzung und die Bewertung schwierig sind: Welche Garantiezusagen sind eine gesonderte Leistung gegenüber dem Verkauf der Aktien und wie soll der Wert solcher Zusagen möglichst belastbar festgestellt werden? ${ }^{35}$

Wie sieht das die Richtlinie? Zunächst stellt Art. 5 Abs. 4 UAbs. 1 ÜbRL auf die Gegenleistung „für die gleichen Wertpapiere“ ab. Das spricht eher für eine engere Auslegung, wonach bei der Ermittlung der erbrachten Gegenleistung formal auf das Rechtsgeschäft abzustellen ist, mit dem die Aktien erworben wurden. Ausschlaggebend ist das aber nicht, denn die Wendung stellt meines Erachtens eher darauf ab, dass bei unterschiedlichen Gattungen von Wertpapieren nur die Gegenleistung für die gleiche Gattung als Referenzpreis dienen soll. ${ }^{36}$ Wichtiger ist die demonstrative Aufzählung der Gründe für Ausnahmen in Art. 5 Abs. 4 UAbs. 2 Satz 2 ÜbRL, wo als ein Grund für die Anpassung genannt wird, dass „der Höchstpreis in einer Vereinbarung zwischen Käufer und Verkäufer gemeinsam festgelegt worden ist“. Das ist zwar sprachlich missglückt, soll aber in der Sache Umgehungssachverhalte erfassen, die gerade den oben genannten Beispielen entsprechen. ${ }^{37}$ Als Ergebnis kann man somit festhalten, dass die ÜbRL davon aus-

31 Näher Tyrolt/Cascante in: Mülbert/Kiem/Wittig (Fn. 8) S. 129.

32 Für Deutschland z.B. Noack in: Schwark/Zimmer (Fn. 27) \31 WpÜG Rn. 29; Reinhardt/Kocher in: Paschos/Fleischer (Fn. 30) \15 Rn. 155.

33 Vgl. die Erläuternden Bemerkungen zum ÜbRÄG 2006, 1334 BlgNR 20. GP, S. 18; Diregger/Kalss/Winner (Fn. 25) Rn. 312.

34 Noack in: Schwark/Zimmer (Fn. 27) §31 WpÜG Rn. 29.

35 Deswegen auch zurückhaltend zur Berücksichtigung solcher Zusagen Reinhardt/ Kocher in: Paschos/Fleischer (Fn. 30) $₫ 15$ Rn. 155; grundsätzlich bejahend aber Krause in: Assmann/Pötzsch/Schneider (Fn. 30) \31 Rn. 114; Kremer/Oesterhaus in: KölnerKomm. WpÜG (Fn. 30) §31 Rn. 71.

${ }^{36}$ Vgl. auch den Bericht der High Level Group (Fn. 7) S. 49, der auf den Preis abstellt, der für "shares in that class“ bezahlt wurde (ähnlich S. 51, 52). Zum Thema auch Hopt, Europäisches Übernahmerecht (Fn. 2) S. 58.

37 Siehe schon oben II. 
geht, dass es nicht um die Ermittlung der Gegenleistung, sondern um die Anpassung des Mindestpreises für das Pflichtangebot geht, wenn Fälle beurteilt werden, in denen Leistungen vom Erwerber an den Veräußerer über andere Rechtsgeschäfte als den Aktienkaufvertrag erbracht werden. Dem entspricht somit die Rechtsprechung des EuGH.

Diese Frage der richtigen Einordnung im Gefüge der ÜbRL ist nicht bloß von akademischem Interesse. Denn zur richtigen Ermittlung der Gegenleistung enthält die Richtlinie keine Vorgaben, zur Anpassung allerdings wie gezeigt sehr wohl. Das hat zumindest zwei Auswirkungen:

Erstens fordert Art. 5 Abs. 4 UAbs. 2 ÜbRL, dass eine ausreichende normative Basis für eine Abweichung gegeben sein muss. Gerade dieser Aspekt war sowohl im Verfahren Periscopus vor dem EFTA-Gerichtshof als auch in Sachen Marco Tronchetti Provera vor dem EuGH zentral; beiden Entscheidungen ist unzweifelhaft $\mathrm{zu}$ entnehmen, dass die Determinierung zwingend ist und trotz des Wortes „können“ in Art. 5 Abs. 4 UAbs. 2 Sätze 2 und 3 ÜbRL den Mitgliedstaaten nicht ins Ermessen gestellt ist. Dieser Aspekt fällt im Ergebnis für Österreich wenig ins Gewicht; denn $₫ 26$ Abs. 3 Satz 1 öÜbG erfüllt meines Erachtens die vom EuGH in Marco Tronchetti Provera aufgestellten - recht liberalen - Anforderungen. Ob die Frage für Deutschland - angesichts der weit gehend durch die Praxis und ohne ausdrückliche Anhaltspunkte im Gesetz konturierten Grundsätze - anders zu beurteilen ist, soll der Fachdiskussion meiner deutschen Kolleginnen und Kollegen überlassen bleiben.

Zweitens ist auch die Anordnung in Art. 5 Abs. 4 UAbs. 3 ÜbRL zu berücksichtigen, wonach die Änderung des Preises auf Basis einer Entscheidung der Aufsichtsbehörde zulässig ist, die begründet sein und bekannt gemacht werden muss. Ob das wirklich in allen Fällen passiert, erscheint mir zweifelhaft, was ich für Österreich kurz darstellen möchte. Gem. \10 Abs. 1 öÜbG hat der Bieter die Angebotsunterlage anzuzeigen; in dieser muss auch der Preis gesetzmäßig ausgestaltet sein. Das bedeutet, dass sämtliche erforderlichen Anpassungen bereits in der Unterlage vorzunehmen sind. Die Übernahmekommission kann sich dann darauf beschränken, die Veröffentlichung der Angebotsunterlage nicht zu untersagen. $\mathrm{Zu}$ einer Entscheidung, die begründet oder veröffentlicht wird, ${ }^{38}$ kommt es nicht.

Das ist meines Erachtens noch unproblematisch, wenn es um eine Anpassung im Sinne einer Erhöhung des Mindestpreises gegenüber der Referenztransaktion geht. Denn dann liegt der Angebotspreis über der formal ermittelten Schwelle, was nach der ÜbRL jedenfalls auch ohne gesonderte Entscheidung der Aufsichtsbehörde zulässig ist. Weigert sich der Bieter,

${ }^{38}$ Natürlich liegt auch der Nichtuntersagung der Veröffentlichung eine Entscheidung der Behörde zugrunde. Diese ergeht aber nicht formell in einer der der Übernahmekommission zur Verfügung stehenden Handlungsformen. 
eine nach Ansicht der Aufsichtsbehörde erforderliche Anpassung vorzunehmen, wird es zu einer Untersagung kommen, die dann jedenfalls begründet sein muss (aber dann auch zu veröffentlichen ist). Heikler ist der umgekehrte Fall, in dem die Anpassung des Mindestpreises nach unten erfolgt, weil z.B. der Verkäufer bestimmte gesondert bewertbare Garantien übernommen hat, deren Barwert von der Referenztransaktion in Abzug gebracht werden soll. Auch hier käme es im Regelfall nach der österreichischen Praxis zu einer Veröffentlichung der Angebotsunterlage, ohne dass eine nach außen tretende Entscheidung der Übernahmekommission vorliegt. Diese Praxis ist angesichts der Rechtsprechung des EuGH zu überdenken. ${ }^{39}$

\section{Wann sind Vorschriften zur Preisanpassung bestimmt genug?}

Damit gelangen wir aber zur zweiten zentralen Frage: Kann den Entscheidungen Periscopus ${ }^{40}$ und Marco Tronchetti Provera ${ }^{41}$ eine gemeinsame Linie entnommen werden oder stehen sie in einem nicht auflösbaren Spannungsverhältnis zueinander? Denn während Periscopus noch strenge Grundsätze an die Determinierung aufstellt und den Verweis nach norwegischem Recht auf den „Marktpreis zum Zeitpunkt, zu dem die Angebotspflicht ausgelöst wird" nicht ausreichen ließ, soll nach Marco Tronchetti Provera eine in Italien gesetzlich angeordnete Anpassung in Fällen der „Kollusion“ ausreichend bestimmt sein. Letzteres ist wohl kaum bestimmter als die norwegische Vorschrift.

Allerdings sind die Ausgangssachverhalte der beiden Entscheidungen unterschiedlich. Denn in Marco Tronchetti Provera ging es um Schutz der Minderheitsaktionäre vor Umgehungen der Mindestpreisregel, wobei diese Umgehungen durch gemeinsame Gestaltungen des Bieters und des Verkäufers eines Aktienpakets herbeigeführt werden. In diesem Zusammenhang ist der Grundsatz der Flexibilität ${ }^{42}$ besonders wichtig. Denn jede kasuistische Aufzählung konkret erfasster Verhaltensweisen müsste sich an bereits beobachteten Praktiken orientieren. Angesichts des Erfindungsreichtums der Praxis könnten neue Gestaltungen nicht adäquat erfasst werden. Will der

39 Dieses formelle Problem kann vermieden werden, indem das Entgelt für eine Garantievereinbarung nicht in den Kaufvertrag über die Aktien, sondern in eine getrennte Vereinbarung aufgenommen wird, weil dann keine gesonderte Anpassung der im Aktienkaufvertrag vereinbarten Gegenleistung (nach unten) erforderlich ist. In der Sache ändert dies an den Beurteilungsmaßstäben freilich nichts.

${ }^{40}$ EFTA-Gerichtshof v. 10.12.2010, Periscopus AS ./. Oslo Bors ASA und Erika Must AS, E-1/10, [2009-2010] EFTA Ct. Rep. 198.

${ }_{41}$ EuGH v. 20.7.2017, Marco Tronchetti Provera SpA et al v Consob, C-206/16, ECLI: EU:C:2017:572. ÜbRL.

Siehe ErwGr (6) ÜbRL und die konkrete Ausprägung in Art. 4 Abs. 5 UAbs. 2 
Gesetzgeber den Umgehungsschutz ernst nehmen und - wie der EuGH zu Recht betont ${ }^{43}$ - den Schutz der Angebotsadressaten, der durch Art. 3 Abs. 1 Buchstabe a ÜbRL zum allgemeinen Grundsatz erhoben wird, erreichen, so muss er notwendigerweise mit unbestimmten Gesetzesbegriffen operieren. Die ausschlaggebende Frage ist dann nur noch, ob diesen Begriffen aus Rechtssicherheitsgesichtspunkten ausreichende Konturen entnommen werden können, was nur der gesamten nationalen Rechtsordnung samt ihren Auslegungsprinzipien entnommen werden kann und damit weit gehend der Entscheidungskompetenz des EuGH entzogen ist. Deswegen ist es auch richtig, wenngleich bei Durchsicht der Entscheidung unbefriedigend, wenn der EuGH in Marco Tronchetti Provera festhält, dass die nationalen Gerichte für die Feststellung zuständig sind, ob der Begriff der Kollusion eine ausreichend bestimmte und voraussehbare Rechtsanwendung zulässt. ${ }^{44}$

Hingegen geht es bei Periscopus um die Frage, ob ein höherer Börsenkurs $\mathrm{zu}$ einer Korrektur des Angebotspreises nach oben führen kann. In diesem Zusammenhang geht es nicht darum, von den Parteien gewählte Gestaltungen normzweckadäquat zu erfassen. Vielmehr knüpft der Gesetzgeber an Marktgegebenheiten an, die durch die Parteien nicht beeinflusst werden können. In diesem Zusammenhang ist es dem Gesetzgeber auch zuzumuten, die genauen Voraussetzungen festzulegen, die eine Anpassung erforderlich machen, wobei der EFTA-Gerichtshof den Beobachtungszeitraum, die Volumensgewichtung und die Frage, ob bloße Orders genügen, explizit anspricht. ${ }^{45}$ Für die Richtigkeit dieses Zugangs spricht auch, dass nationale Rechtsordnungen, die Börsenkurse überhaupt berücksichtigen, in der Regel solche Determinierungen enthalten, so auch $₫ 5$ WpÜG-AngVO und $₫ 26$ Abs. 1 Satz 3 öÜbG.

Damit kann man für das Spannungsverhältnis von Flexibilität und Vorhersehbarkeit in Zusammenhang mit der Preisbildung als allgemeinen Grundsatz ableiten, dass das Gebot der Determinierung gem. Art. 5 Abs. 4 UAbs. 2 ÜbRL situationsspezifisch auszulegen ist: Die Determinierung muss umso konkreter sein, je eher die Anpassung an objektiven Faktoren festgemacht wird, die von den Parteien nicht beeinflusst werden können. Geht es allerdings darum, zum Schutz der austrittsberechtigten Gesellschafter von den Parteien gewählte Gestaltungen zu erfassen, genügt es, wenn der Gesetzgeber generelle Prinzipen festlegt, die dann im Einzelfall durch Auslegung konkretisiert werden müssen.

${ }^{43}$ EuGH v. 20.7.2017, Marco Tronchetti Provera SpA et al v Consob, C-206/16, ECLI: EU:C:2017:572, Rn. 33, 45.

${ }_{44} \mathrm{AaO}$ Rn. $46 \mathrm{f}$.

45 EFTA-Gerichtshof v. 10.12.2010, Periscopus AS ./. Oslo Bors ASA und Erika Must $A S$, E-1/10, [2009-2010] EFTA Ct. Rep. 198, Rn. 49. 


\section{Der Börsenkurs}

Zum Abschluss ist auf die Frage des Börsenkurses als zweite Preisuntergrenze einzugehen. In der Rechtssache Periscopus stand eine solche Regelung im Mittelpunkt, wobei jedoch nur die konkrete Ausgestaltung der Regelung in Zweifel gezogen wurde, nicht aber die grundsätzliche Frage angesprochen wurde, ob es überhaupt zulässig ist, den Börsenkurs als zweite Preisuntergrenze heranzuziehen. Allerdings könnte es diesbezüglich in absehbarer Zeit Neuerungen geben, da im Rahmen eines lettischen Vorabentscheidungsersuchens ${ }^{46}$ unter anderem ${ }^{47}$ die Frage gestellt wird, ob eine Regelung richtlinienkonform ist, wonach der Mindestpreis des Pflichtangebots jedenfalls dem gewichteten durchschnittlichen Börsenkurs innerhalb der letzten zwölf Monate vor dem Entstehen der Angebotspflicht entsprechen muss. Konkret ist derzeit ${ }^{48}$ noch wenig über das Ersuchen bekannt, da es erst im Oktober 2019 eingelangt ist. Allerdings ist es Anlass, die Frage der Richtlinienkonformität einer solchen Regelung, wie sie ja auch $₫ 5 \mathrm{WpÜG-}$ AngVO und $₫ 26$ Abs. 1 Satz 3 öÜbG vorsehen, zu beleuchten.

Traditionell wird die Zulässigkeit solcher Vorschriften damit begründet, dass die ÜbRL bloß mindestharmonisierend wirkt: Gem. Art. 3 Abs. 2 Buchstabe $b$ ÜbRL sind zusätzliche Bedingungen und strengere Bestimmungen für Angebote als in der Richtlinie zulässig, was nach ganz überwiegender Ansicht unter anderem zusätzliche Preisvorschriften erfasst. ${ }^{49}$

Aber auch in diesem Zusammenhang stellt sich die Frage, ob diese Einordnung wirklich richtig ist. Denn genauso gut lässt sich die Berücksichtigung des Börsenkurses als Anpassung des Vorerwerbspreises verstehen. Dies ist auch das Verständnis des EFTA-Gerichtshofs in der Rechtssache Periscopus, in der die Zulässigkeit der norwegischen Regelung, nach der welcher der deutlich höhere Marktpreis, also der Börsenkurs, maßgeblich sein sollte, gerade nicht Art. 3 Abs. 2 Buchstabe b ÜbRL unterstellt, sondern an den Vorgaben von Art. 5 Abs. 4 UAbs. 2 ÜbRL gemessen wurde.

46 C-735/19 Euromin Holdings (Cyprus) Limited v Finanšu un kapitāla tirgus komisija.

47 Daneben geht es auch um die Fragen, ob ein höherer Wert des Nettoaktivvermögens ausschlaggebend sein darf, wie ein solcher Wert konkret berechnet werden muss und ob aus Richtlinienverletzungen Schadenersatzpflichten der Mitgliedsstaaten entstehen können.

48 Dezember 2019.

49 Vgl. z.B. Lutter/Bayer/J. Schmidt (Fn. 14) Rn. 28.55; Kalss/Klampfl in: Dauses, Handbuch des EU-Wirtschaftsrechts, Loseblatt, E.III., Rn. 515; Krause in: Assmann/ Pötzsch/Schneider (Fn. 30) § 31 Rn. 22; Stiegler in: Jung/Krebs/Stiegler, Gesellschaftsrecht in Europa, 2019, \$29 Rn. 57. Für Österreich vgl. die Erläuternden Bemerkungen zum ÜbRÄG 2006, 1334 BlgNR 20. GP, S. 18; Diregger/Kalss/Winner (Fn. 25) Rn. 304; Huber in: Huber (Fn. 26) \& 26 Rn. 5. AM z.B. Maul/Muffat-Jeandet, AG 2004, 221, 230 f. 
Das ist meines Erachtens auch konsequent. Denn die Ausnahmevorschrift in Art. 5 Abs. 4 UAbs. 2 ÜbRL ist als gesonderte Vorschrift für die Preisbildung bei Pflichtangeboten ${ }^{50} \mathrm{zu}$ verstehen, die vor anderen Bestimmungen der Richtlinie Vorrang hat. Dieser Charakter als lex specialis ist für die Generalausnahmeklausel in Art. 4 Abs. 5 UAbs. 2 ÜbRL anerkannt. Darüber hinaus spricht aber der Regelungszusammenhang von Art. 5 Abs. 4 UAbs. 2 ÜbRL dafür, dass die Richtlinie bezüglich der Preisvorschriften vollharmonisierend wirkt; denn sonst wäre eine strengere Vorschrift nicht am Determinierungsgebot dieser Norm zu messen - mit anderen Worten hätte sich die im Periscopus-Verfahren untersuchte Frage, ob die norwegische Norm determiniert genug ist, bei der Richtigkeit der in Deutschland und Österreich herrschenden Ansicht überhaupt nicht ${ }^{51}$ gestellt. Der Gesamtkontext spricht somit dafür, dass Art. 5 Abs. 4 UAbs. 2 ÜbRL Anpassungen des Mindestpreises im Vergleich zur Vorerwerbsregel abschließend regelt und damit auch die deutsche und österreichische Börsenkursregel erfasst. ${ }^{52}$

Dem kann meines Erachtens auch nicht entgegengehalten werden, dass nach dem Wortlaut von Art. 5 Abs. 4 UAbs. 2 ÜbRL nur Anpassungen durch die Behörde selbst erfasst sind, die deutsche und österreichische Börsenkursregeln aber gesetzliche Anpassungen enthalten, die automatisch, d.h. ohne jegliche Tätigkeit der Behörde anwendbar sind. Denn wie die jeweilige nationale Norm konkret ausgestaltet ist, kann für die Anwendung der Richtlinien-Vorschrift nicht entscheidend sein. Ausschlaggebend ist nur, dass die Abweichung vom maßgeblichen Erwerbspreis letztlich durch die Behörde (oder durch ein Gericht) durchgesetzt werden kann. Wäre dies anders, so wäre eine Norm, nach der die Behörde bei höherem Börsenkurs den Angebotspreis durch Entscheidung anpassen muss, von Art. 5 Abs. 4 UAbs. 2 ÜbRL erfasst, eine andere Norm, bei der dieselbe Anpassung nach dem gesetzlichen Konzept durch die Parteien selbst, aber vor dem Hintergrund behördlicher Sanktionen erfolgt, hingegen nicht. Das Ergebnis ist aber in beiden Fällen dasselbe: Der höhere Börsenkurs ist maßgeblich. Auch Fälle wie die Periscopus-Entscheidung, in denen die Anpassung grundsätzlich von Gesetzes wegen erfolgt, unterliegen damit Art. 5 Abs. 4 UAbs. 2 ÜbRL.

Misst man solche Vorschriften an den Vorgaben von Art. 5 Abs. 4 UAbs. 2 ÜbRL, so kann man dies ohne weiteres wie folgt tun: Der deutsche und der österreichische Gesetzgeber haben entschieden, dass unter bestimmten Voraussetzungen ein höherer Marktpreis zu berücksichtigen ist, also eine Anpassung der Mindestgegenleistung nach oben vorgenommen

${ }^{50}$ Nicht bei Übernahmeangeboten zur Kontrollerlangung, auf die Art. 5 ÜbRL nicht anwendbar ist.

51 Oder jedenfalls nicht auf Ebene der Auslegung der ÜbRL.

52 Ausdrücklich anders aber Lutter/Bayer/J. Schmidt (Fn. 14) Rn. 28.55 bei und in Fn. 255. 
wird. Das ist der Fall, wenn die näheren Voraussetzungen nach $₫ 5 \mathrm{WpÜG-}$ AngVO bzw. \26 Abs. 1 ö̈̈bG vorliegen; diese sind im Sinne der Periscopus-Entscheidung für Zwecke von Art. 5 Abs. 4 UAbs. 2 ÜbRL ausreichend determiniert. Anwendbar ist dann der solcherart ermittelte gewichtete durchschnittliche Börsenkurs der letzten drei (Deutschland) bzw. sechs (Österreich) Monate - eine Möglichkeit, die in Art. 5 Abs. 4 UAbs. 2 Satz 3 ÜbRL ohnehin ausdrücklich genannt wird. Es stört nicht, dass die Voraussetzung für die Abweichung und die Kriterien, mit denen dann der Mindestpreis bestimmt wird, gleich sind. Einer begründeten und veröffentlichten Entscheidung im Sinne von Art. 5 Abs. 4 UAbs. 3 ÜbRL bedarf es im Übrigen auch in diesem Zusammenhang ${ }^{53}$ nicht, sofern der Bieter die entsprechende Anpassung selbst vornimmt.

Es ist somit nicht davon auszugehen, dass Regelungen zur Maßgeblichkeit des Börsenkurses ${ }^{54}$ wie die lettische, deutsche oder österreichische den Vorgaben der Richtlinie widersprechen. Das ist unstrittig, wenn man die Zulässigkeit mit der herrschenden Meinung auf die Möglichkeit stützt, strengere Vorschriften vorzusehen, gilt aber auch bei der hier vertretenen Subsumtion unter Art. 5 Abs. 4 UAbs. 3 ÜbRL, die lediglich den Prüfmaßstab, nicht aber das konkrete Ergebnis ändert. Dieses Ergebnis entspricht im Übrigen auch der Tatsache, dass bereits vor In-Kraft-Treten der ÜbRL viele Mitgliedstaaten die Maßgeblichkeit von Börsenkursen vorsahen, die Kommission dem - in Kenntnis dieser Vorschriften ${ }^{55}$ - aber keinen Riegel vorgeschoben hat.

\section{Schluss}

Bereits 2002 hat der Bericht der High Level Group, der äußerst einflussreich für die endgültige Ausgestaltung der ÜbRL war, unter Beteiligung von Klaus J. Hopt die wesentlichen Faktoren für eine angemessene Regelung des Mindestpreises identifiziert: ${ }^{56}$ Respekt für die Regelungstraditionen der Mitgliedstaaten, ein vernünftiger Grad an Flexibilität bei der Anwendung der Regelungen und eine ausreichende Vorhersehbarkeit des letztlich zu bietenden Preises. An der Maßgeblichkeit dieser Faktoren hat sich seitdem nichts geändert.

Ebenso wenig ist zu erkennen, dass Art. 5 Abs. 4 ÜbRL grundsätzlich zu überarbeiten wäre. Gerade die Rechtsprechung des EuGH in diesem Be-

53 Zur parallelen Frage bei verschleierten Gegenleistungen siehe oben III.B.

${ }^{54}$ Das muss aber grundsätzlich auch für Vorschriften gelten, welche einen höheren Unternehmenswert für maßgeblich erklären, was auch Teil der Vorlagefragen des lettischen Gerichts ist und für $\$ 5$ Abs. 4 WpÜG-AngVO Bedeutung haben kann.

${ }_{55}$ High Level Group (Fn. 7) S. 47.

${ }^{56}$ High Level Group (Fn. 7) S. 49. 
reich zeigt, dass diese Regeln das - letztlich nie vollkommen auflösbare Spannungsverhältnis zwischen Flexibilität bzw. Einzelfallgerechtigkeit einerseits und Rechtssicherheit andererseits ausreichend gut bewältigen. Dass die Rechtslage in den Mitgliedstaaten unterschiedlich ist, darf auch nicht als Manko betrachtet werden - jedenfalls solange der Nachweis nicht gelingt, dass es für die Frage des Mindestpreises eine ökonomisch richtige Lösung gibt, die nicht von den konkreten Gegebenheiten in den einzelnen Mitgliedstaaten abhängig ist. Es bleibt zu hoffen, dass auch der EuGH dieses Bekenntnis zur Vielfalt teilt. Jedenfalls bleibt die Ansicht des Jubilars gültig: Es gibt keinen Grund die Preisbildungsvorschrift der ÜbRL zu überarbeiten. 
